\title{
Heuristically Semi-automated Segmentation of Femur from 3-D CT Images
}

\author{
Zhiwei Huang, Ning Dai, and Hao Liu
}

\begin{abstract}
It's hard to segment CT slices featured contour accurately and rapidly. As we all know, the segmentation of femur contour is an essential operation for the medical image 3D reconstruction, most of the software to extract the CT contour layer by layer, which is not only time-consuming but also inaccurate. According to the limitation, this paper comes up with a semi-automatic segmenting method for femur contour, which is based on heuristic. To start with, we enhance all the edges of the image. Secondly, a new method which is based on heuristic contour extracted technology is used to extract slice sequence outlines. Semi-automatic contour extractive experiments with CT slice data verify the validity and practicability of the algorithm.
\end{abstract}

Index Terms-CT slices, outline extraction, snake algorithm.

\section{INTRODUCTION}

Owing to the development of computer graphics and the wide application of image processing technology, the new methodologies can be applied to diagnose lesions [1], [2]. The wide application of CT images can be concluded as the following four aspects [3]: 1) CT slice3D reconstruction for surgical planning and postoperative evaluation; 2) data displaying and feature extraction make doctors observe lesions are as more intuitively; 3) surgery simulates multi-angle reorganization and original data simulates multi-angle scan; 4) accurate locating radiotherapy can reduce the dose of radiation and the damage to human body.

Due to a layer of smooth cartilage with low density and blood vessels covering the head of femur, it leads to the artifacts and the diffused texture of the joint between femur and pelvis in CT images. What's worse, the scope of femoral outline shape changes dramatically. Therefore, the methods described in other papers [4]-[6] and used in the liver and lung contour extraction [7] are difficult to be applied to extract femur contour. Reza A. Zoroofi [8] came up with a new methodology, which, firstly, sets the optimal threshold with histogram, then filtering and combining the moving disk technology to extract the femur contours. It is an effective technique to achieve the ideal results, but the efficiency should be improved.

After analyzing the advantages and disadvantages of the

Manuscript received October 20, 2014; revised February 12, 2015. Thi work was supported by the National Natural Science Fund of China (51205192), the National High Technology Research and Development Program863 (SS2013AA040801) and supported by the Fundamental Research Funds for the Central Universities (NJ20130015, NZ2013201).

The authors are with the College of Mechanical and Electrical Engineer, Nanjing University of Aeronautics and Astronautics, Nanjing, China (e-mail: hzw1003613248@126.com,_dai_ning@nuaa.edu.cn, liuhao-01@nuaa.edu.cn). above extraction methods, a new CT image contour semi-automatic extraction technology is proposed, which is based on heuristic principle. In the beginning, we manually pick up the first layer of femur section contour and load it into the initial contour list. Then the initial contour is mapped to the next layer. According to the one-to-one matching relationship, we use the original outline to approach the next layer contour step by step by using the snake algorithm. Get the next layer of contour and load it into initial contour list. In the same way, we map the outline to the next layer. According to the coordinates of corresponding points and their normal vectors, the algorithm fits discrete contour edge pixels with the initial outline.

The result of our experiments prove that the algorithms can not only meet the requirements of medical diagnosis in practice but also they have a great contribution to the improvement of speed and quality in digital medical diagnosis and treatment. Semi-automatic extraction of CT data contour can improve the efficiency and success of the surgery largely, which is the tendency of digital medical development in the future.

\section{PROCEDURE FOR PAPER SUBMISSION}

There are four procedures to extract outlines of CT date semi-automatically and accurately. The first procedure is to apply the slice contour enhancement technology on the boundaries that have artifacts and diffused texture. The following procedure is to pick up characteristic area contour interactively as the first outline of heuristic semi-automatic segmentation. The third procedure is to pass the first contour to the next layer as the initial outline of snake algorithm. Last but not least, the initial outline approaches the featured area discrete pixels gradually. Before using snake algorithm, we should calculate maximum curvature points on the original curve. Then we structure the control polygon with the curvature points. Specific technical route is shown in Fig. 1.

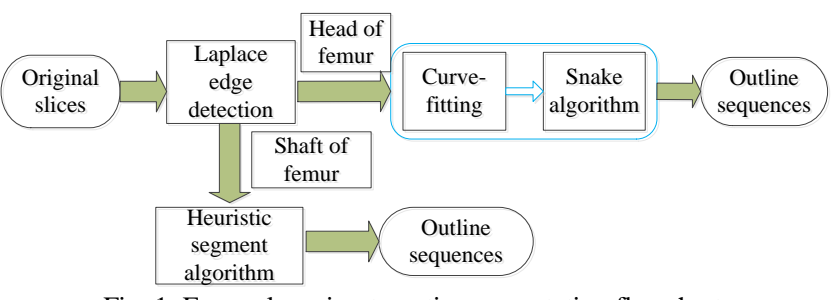

Fig. 1. Femoral semi-automatic segmentation flowchart.

\section{A. Enhancing the Boundary}

The outline of upon layer CT slice is difficult to recognize and segment. So it is essential to take some measures to 
enhance the boundary. There are a lot of algorithms to enhance the outlines, such as Laplace operator, Sobel operator, Canny operator and so on. Our method is different from the traditional methods. As we all know, femoral head is covered with a layer of smooth cartilage and blood vessels. At the same time, the constant friction at the femoral head produces the artifact and diffused texture on the border area of CT slices which makes the femoral contour become more difficult to be extracted. As our outline segmenting process is based on first outline of CT slice. In the beginning, it is important to manually pick up the first layer of femur section contour and load it into the initial contour list. The list space was built before the segmenting process. Then the first outline is mapped to the next layer. According to the one-to-one matching relationship, we use the original outline to approach the next layer contour step by step by using the snake algorithm. Get the next layer of contour and load it into initial contour list. From above process, we can conclude that in beginning of the segmentation, it is important to get the enhanced boundaries. This paper proposes a kind of CT image enhancement algorithm. Firstly, one of the edge detection methods is used to detect boundary contour brightness gradient of the CT slice. Then we combine bright gradient outline with the original CT slice to form a CT image with the boundary enhanced. Compared with other several kinds of edge detection operators, Laplace operator not only can preserve the normal vectors of the inside and outside edge pixels but also the computational efficiency and boundary extraction accuracy are much higher than other kinds of operators. From all above, this paper chooses the Laplace operator to enhance all the boundaries.

\section{B. Femoral Slice Segmentation Process}

Due to a layer of smooth cartilage with low density and blood vessels covering the head of femur, it leads to the artifacts and the diffused texture of the joint between femur and pelvis in CT images. It is hard to segment the slice areas directly. So in the beginning, we should segment the first layer's outline manually. Exactly, when the upper contour is difficult to map to the next layer. We always need to segment the slices with hand.

Two neighboring slices of femur contours are similar both in shape and position. The principle of similar inheritance proposed in this paper is the foundation of heuristic femur section semi-automatic segmentation. The specific process is as follows:

In the beginning, we manually pick up the first layer of femur section contour and load it into the initial contour list. Then the initial contour is mapped to the next layer. According to the one-to-one matching relationship, we use the original outline to approach the next layer contour step by step by using the snake algorithm. Get the next layer of contour and load it into initial contour list. In the same way, we map the outline to the next layer. According to the coordinates of corresponding points and their normal vectors [9], the algorithm fits discrete contour edge pixels with the initial outline.

Fig. 2(a), slice of the femur $T_{n}$, its outline $B_{n}$ maps to the next layer of slice $T_{n+1}$ (Fig. 2(b)). We use the snake algorithm to approach $B_{n+1}$ and generate fitting outline $B_{n, n+1}$ (shown in Fig. 2(c)). In the same way, the contour $B_{n, n+1}$ maps to the next layer as the initial contour. Then it closes to $B_{n+2}$ and generates $B_{n+1, n+2}$ (as shown in Fig. $3)$. Finally, we realize the heuristic contour extraction.

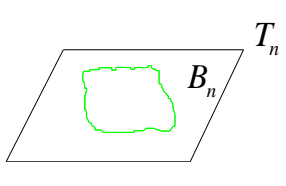

(a) $n$th layer of contour

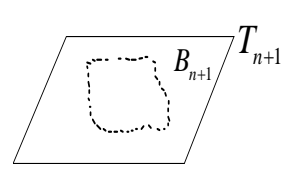

(b) $(n+1)$ th pixel contour

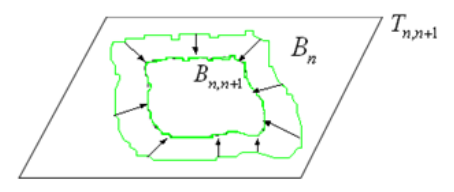

(c) Fitting outline

Fig. 2. Approaching the pixel contour.

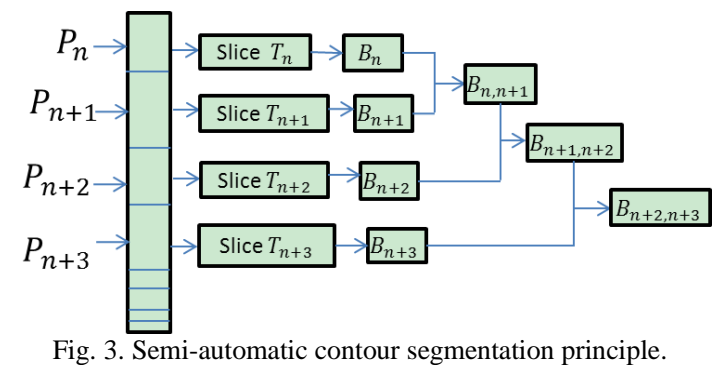

\section{The Snake Algorithm}

Snake algorithm, shrink-wrapping algorithm [10] and the deformation algorithm are similar. Firstly, we should introduce the concept of shrink-wrapping algorithm. The physical model of the process of shrink wrapping is that a plastic membrane is wrapped around an object and shrunk either by heating the material or by evacuating air from the space between the membrane and the object's surface. At the end of this process, the plastic skin provides an exact imprint of the given geometry. The concept of snake or deformable surfaces can be regarded as a simulation of the shrink wrapping process. During the process each vertex is moved according to a force applied to it. The force is a combination of two components. One component is pulling each vertex in the direction of the given surface and the other component is pushing the vertices in order to minimize the local distortion energy within the membrane. The relaxing force supports the distribution of the vertices over the surface. In order to make the initial outline approximate the discrete pixel contour accurately, the snake algorithm is extended to CT data in this paper. We approach the extracting contour pixels gradually by adjusting the initial control points on the initial contour.

If the upper outline surrounds the next layer of contour completely, the snake algorithm will be used inward, vice versa. However, when the outlines intersect with each other, we cannot determine contracting direction inward or outward directly. In this paper, we apply the threshold method to determine the direction of contraction outline and 
get the discrete pixels that meet the demands. It is necessary to establish the one-to-one relationship between discrete points and the curvature maxima points on the outline. The common method is the closest point projection algorithm. The algorithm provides us a method to calculate the shortest distance from discrete point $p_{i}$ to the initial point $q_{i}$ that is one of the points on the outline.

\section{EXPERIMENTAL RESULTS AND DISCUSSION}

We use the VS2008 software as the developing tool and OpenGL 2.0 as image rendering tool. At the same time, we build the soft framework by using the MFC class libraries. This paper mainly takes the experiment on the femur CT tomography image sequence. We use the algorithm in this paper semi-automatically extract the contours of the left femur. The results are shown in Fig. 5. Besides, we compared our result with that of Commercial Mimics software manual segmentation (shown in Fig. 4).

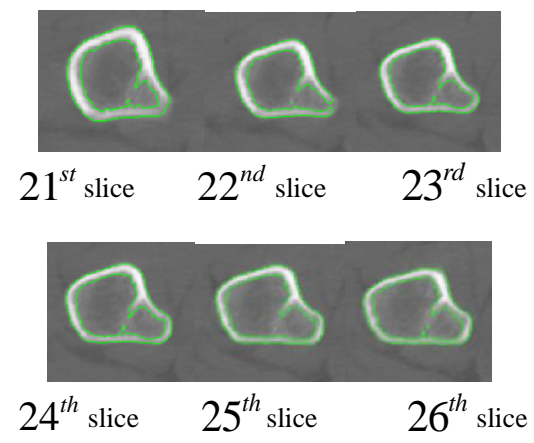

Fig. 4. Contour extracted with Mimics software.
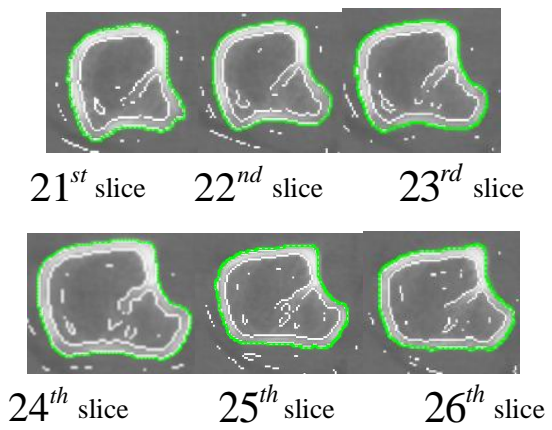

Fig. 5. Contour extracted with our algorithm.

From Fig. 4 and Fig. 5 we can learn that extracting the contour boundary with Mimics software has the drawback of regional rupture, due to the unreasonable threshold setting. The fracture can be avoided by extracting outline with snake algorithm. Because we extract the first layer of femur section contour and load it into the initial contour list. Then the initial contour is mapped to the next layer. The snake algorithm can make sure that every outline is a closed curve.

It illustrates that the three-dimensional femur model that is reconstructed with our algorithm is similar to the model that from the commercial software. It takes a long time to extract the femur CT contour layer by layer and build 3D model with Mimics software, while the time for heuristic outline semi-automatic extraction algorithm is much less. It is proved that the algorithms can not only meet the requirements of medical diagnosis in practice but also they have a great contribution to the improvement of speed in digital medical diagnosis and treatment.

The comparison of the results between our algorithm with semi-automatic segmentation and Mimics commercial software with manual segmentation, the comparison parameters as below:

1) $\bar{T}$ : The average time to segment femur CT tomography image sequence with hand by the Mimics software.

2) $t:$ The average time to segment femur CT tomography image sequence by using our algorithm

3) $P_{n}:$ The evaluation indicator between semi-automatic segment outlines and the real outlines, the specific meaning is shown in (1).

$$
P_{n}=\frac{\sum_{i=1}^{N}\left(\left|\bar{d}_{i}-\bar{d}\right|\right)}{N}
$$

The parameter $\bar{d}_{i}$ represents the average distance from the outline points to the central points on the $i$ th CT tomography image sequence with our algorithm. $\bar{d}$ represents the real average distance from the outline points to the central between the $i$ th CT tomography image sequence. The specific meaning of the parameter is shown in (2), The parameter $\Delta d$ represents the average error distance.

$$
\Delta d=\left|\bar{d}_{i}-\bar{d}\right|
$$

The average time of our algorithm to segment the same tooth contour is much less than that of Mimics commercial software, it improves the efficiency greatly, at the same time, the average error of the real outline and the segmented outline is nearly. We take a series of experiments to verify our algorithms. The result of our experiments prove that the algorithms can not only meet the requirements of medical diagnosis in practice but also they have a great contribution to the improvement of speed and quality in digital medical diagnosis and treatment.

\section{CONCLUSION}

With the improvement of the computer operating ability and the development of medical image aided diagnosis technology, it makes great changes in disease diagnosis and treatment process. Semi-automatic extraction of CT data contour and $3 \mathrm{D}$ reconstruction can improve the efficiency and success of the surgery largely, which is the tendency of digital medical development in the future.

Due to the shortcomings of the traditional CT data extraction. A new contour segmentation method of DICOM data is proposed. As we all know that it is hard to segment femur contour of CT slices automatically and precisely now. In this paper we initially come up with an effective algorithm, which is based on the concept of inherited segmentation intelligently. In order to realize extracted slice 
pass down to the next layer automatically, initial contour is achieved by inheritable mapping. In the paper, the algorithm that is usually applied in surface subdivision is used to extract pixels contour. It not only can realize semi-automatic segmentation slice contour but solve the flaw of fracture. The size of femur for each patient is a far cry from others. The femoral mutations need more experimental data to evaluate. In the future, our experiment will pay more attention to the aspect.

\section{ACKNOWLEDGEMENT}

The authors would like to thank the following people for their help in preparing this work: Bai Xu for his help in preparing data and images, Yi-hao Duan for manual segmentation of the femur slices and sharing information with us. I appreciate deeply for my supervisor who always helps me and supports my work and appreciate for the support from the College of Mechanical and Electrical Engineering, Nanjing University of Aeronautics and Astronautics.

\section{REFERENCES}

[1] T. Sun and D. Q. Gao, "3D reconstruction medical ct image in individualized bone," Machinery Design \& Manufacture, vol. 9, no. 2, pp. 262-263, 2010

[2] X. T. Jiang, L. M. Luo, and J. W. Wang, "An automatic segmentation approach for CT serial images lung tumors," Journal of Image and Graphics, vol. 9, no. 8, pp. 1028-1033, 2003.

[3] X. Li, Research and Application on CT Image, Harbin Engineering University, 2004

[4] X. L. Hou, B. L. Wang, S. H. Huang, and X. Song, "Automatic segmentation of serial CT liver images based on fast marching method," China Digital Medicine, vol. 4, no. 2, pp. 32-35, 2008.

[5] P. Jiao, J. Roos, A. Chin et al., "Adaptive border marching algorithm: Automatic lung segmentation on chest CT images," Computerized Medical Imaging and Graphics, vol. 32, no. 6, pp. 452-462, 2008.

[6] F. Zhang, Y. B. Fan, F. Pu, and Z. Liu, "A semi-automatic method for tooth segmentation in dental CT images," Journal of Biomedical Engineering, vol. 24, no. 1, pp. 15-18, 2007.
[7] M. Ciecholewski, "Automatic liver segmentation from 2D CT images using an approximate contour model," Journal of Signal Processing System, vol. 74, no. 10, pp.151-174, 2014.

[8] R. A. Zoroofi, Y. Sato, T. Sasama et al., "Automated segmentation of acetabulum and femoral head from 3-D CT images," IEEE Transactions on Information Technology in Biomedicine, vol. 7, no. 4, pp. 329-343, 2003.

[9] N. Dyn, D. Leven, and J. A. Gregory, "A 4-point interpolatory subdivision scheme for curve design," Computer Aided Geometric Design, vol. 4, no. 4, pp. 257-268, 1987.

[10] L P. Kobbelt, J. Vorsatz, U. Labsik et al., A shrink wrapping approach to remeshing polygonal surfaces. [Online]. Available: http://www.graphics.rwth-aachen.de/media/papers/remesh1.pdf

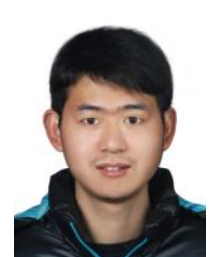

Zhiwei Huang was born in Anhui, China in 1988. He received his bachelor degree in mechanical design manufacturing and automation in Tong Ling College, China. He is currently working towards his master degree in the College of Mechanical and Electrical Engineering Nanjing University of Aeronautics and Astronautics, Nanjing, China. His research interests are computer graphics, biomedical engineering technology, and discrete data processing.

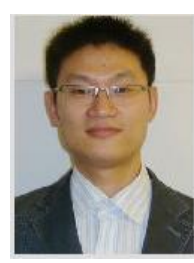

Ning Dai received a doctorate from the College of Mechanical and Electrical Engineering of Nanjing University of Aeronautics and Astronautics Nanjing, Jiangsu, China in 2005. He now is an associate professor, engaging in research and teaching work in Nanjing University of Aeronautics and Astronautics. His main research interests are CAD/CAM and computer graphics, biomedical engineering technology, discrete data processing.

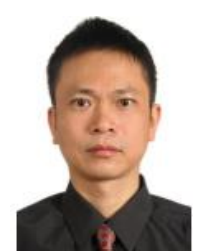

Hao Liu received a doctorate from the College of Mechanical and Electrical Engineering of Nanjing University of Aeronautics and Astronautics in 2005. He now is an associate professor, engaging in research and teaching work in Nanjing University of Aeronautics and Astronautics. His main research interests are $\mathrm{CAD} / \mathrm{CAM}, \mathrm{CAGD}$ and image processing. 